\title{
PIEZOELECTRIC PLASTIC MICROPHONES ${ }^{\dagger}$
}

\author{
J.A.GRUBB \\ Plessey Connectors Limited, Northampton \\ Received 26th October 1981
}

\begin{abstract}
Current types of microphones are described and compared with designs based on a bimorph diaphragm made from piezoelectric poly(vinylidene fluoride) film. A pressure operated microphone for telephone handsets has been fully developed. Polymer film diaphragms lend themselves to highly symmetrical designs so that both first and second order pressure gradient noise cancelling units have been based on them and compare well with the available alternatives.
\end{abstract}

\section{INTRODUCTION}

\section{A Survey of Microphones}

Since the invention of the telephone over a century ago a wide range of microphones has been developed for various specific purposes. The world's telephone systems have been, and still are, based on the carbon granule microphone (see Figure 1) invented by Hunnings in 1878 (ref. 1). In this device, the sound impinges on a diaphragm and the resulting vibrations are conveyed to a carbon button and thence to carbon granules placed between this and another button. The resistance of the granules is modulated by the vibration so that if a constant current is passed through the microphone a varying voltage appears across it. This type of microphone has a high sensitivity compared to other types and is the only microphone which can be used without any amplification in a telephone system. However, it requires a high current (typically $50-100 \mathrm{~mA}$ ) and is prone to deteriorate with time and usage. It is sensitive to movement and tends to produce rustling noises as its position is changed. It is robust and will withstand mechanical and electrical abuse without failing completely. Being a non-linear device, it is not suited to high-quality applications, and is seldom encountered elsewhere than in telephones.

Two types of microphones rely upon the induction of a voltage into a conductor by a changing magnetic flux. In one case, the moving-iron microphone (Fig. 2) a magnetic diaphragm may be vibrated by incident sound in close proximity to a coil. Alternatively, the conductor may itself be moved in a stationary magnetic field, as in the moving-coil microphone (Fig. 3) where the conductor is in the form of a light cylindrical coil which is attached to a domed diaphragm. In the ribbon microphone, the conductor and diaphragm are combined in the form of an extremely thin corru- gated aluminium ribbon, which is sited between the poles of a magnet. The ribbon microphone can provide extremely high quality signals but is heavy and also delicate because of the fragility of the ribbon. It tends to be used mainly in recording and broadcasting studios. The moving coil microphone can provide a fairly high quality signal, although not as high as a ribbon, and an extended frequency range, although this necessitates the use of a very light coil and diaphragm.

Moving-coil and moving-iron microphones both use coils of fine wire and moving parts separated by very small clearances with the attendant complications in manufacture. Moving-iron microphones are used as noise-cancelling microphones but it is difficult to make such a microphone respond equally to front and rear sounds over an extended frequency range. The importance of this symmetry is discussed in the section on noise-cancelling microphones.

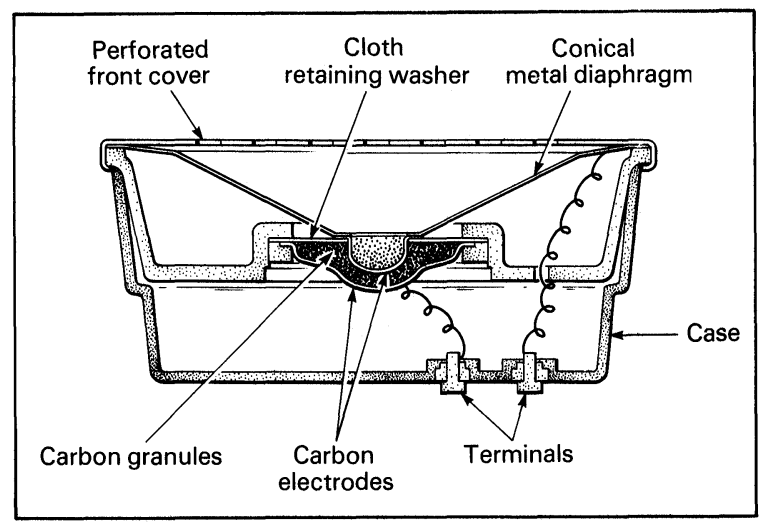

FIGURE 1 Carbon Granule Microphone - section through centre.

† Originally published in the 1980 Annual Review of the Allen Clark Research Centre and reprinted by permission of the Plessey Co. 


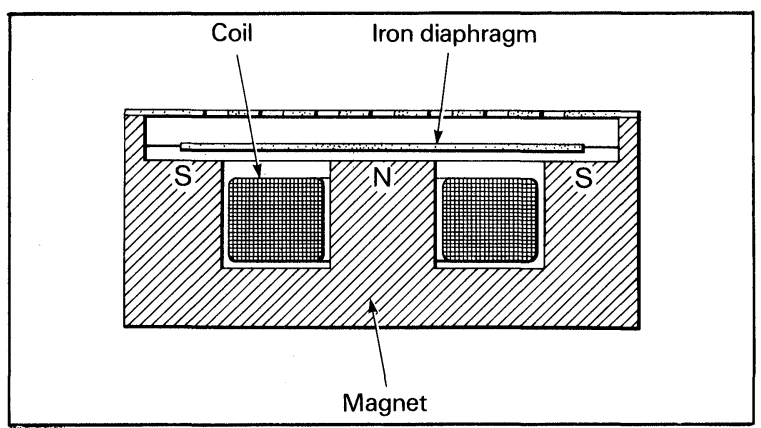

FIGURE 2 Moving-Iron Microphone

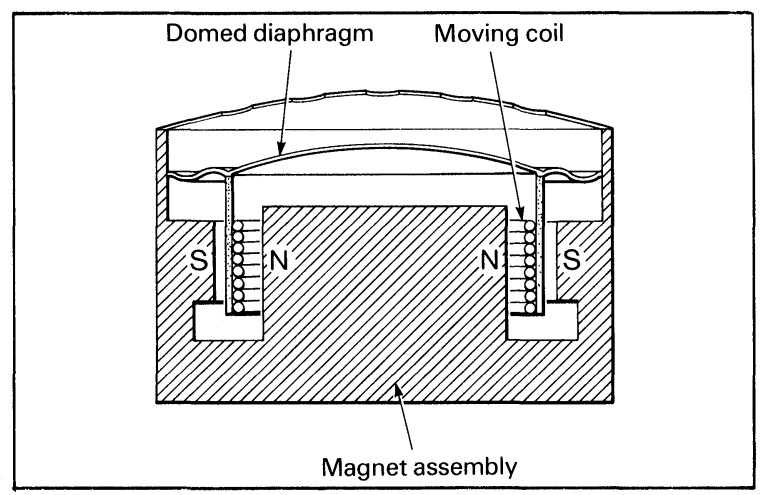

FIGURE 3 Moving-Coil Microphone

Capacitor microphones (Fig. 4) are constructed as parallel-plate capacitors, in which a thin conducting diaphragm is placed very close to a fixed, rigid backplate. An electric field is applied across the gap by means of an external dc supply (100-200V), or a permanently polarised electret, and as the diaphragm vibrates the change in capacitance gives rise to a change in voltage.

Externally polarised capacitor microphones provide the highest quality performance of all. It is possible to make a capacitor microphone with a level response from below $1 \mathrm{~Hz}$ to above $40 \mathrm{kHz}$, and with the sensitivity stable to better than $1 \mathrm{~dB}$ over several years at room temperature. Because of this, the capacitor microphone is universally used for acoustic measurements. It is also possible to make directional capacitor microphones, for instance the cardioid, which has maximum sensitivity on axis and ideally zero response to sound from the rear. The directional response can be maintained accurately over a wide frequency range which is important in stereo and multi-channel recording. For these reasons, capacitor microphones are widely used for recording and broadcasting. The electret is a further development of the capacitor microphone in which the metal diaphragm is replaced by one of metallised plastic, commonly fluorinated ethylene propylene (F.E.P.), which has been poled by the application of an electric field and high temperature. The material retains an electric dipole moment and this obviates the need for an external polarising supply. Since the inner side of the diaphragm is non-conducting there is no need to ensure that it does not touch the backplate and therefore electret microphones are often made using a backplate which has a series of raised dimples over which the diaphragm is laid and which set the capacitor gap spacing. The construction also relaxes the restrictions on diaphragm tension which are critical for good performance in a classical capacitor microphone. To obtain good sensitivity, the capacitor gap must be small, typically $25 \mu \mathrm{m}$ and consequently these microphones must be assembled in clean conditions.

Both types of capacitor microphone form a source with a very low internal capacitance $(\sim 10-50 \mathrm{pF})$ and therefore require an amplifier in close proximity. The amplifier must have a high input impedance, in some measuring microphones in excess of 4000 megohms, to avoid low frequency losses. Because of these high impedances, capacitor microphones are susceptible to moisture and precautions are usually needed to avoid condensation when they are used for close talking or in humid conditions. Some measuring microphones have a heater built in to the amplifier housing for this purpose. Condensation on the capsule insulator will probably produce high background noise and excessive leakage may result in the destruction of the amplifier input transistor. Electret microphones are used in consumer equipment and sometimes for recording and broadcasting.

Microphones using piezoelectric crystals and ceramics were widely used in consumer equipment some years ago but have now been largely superseded by electrets. Figure 5 shows a cross section of a cera-

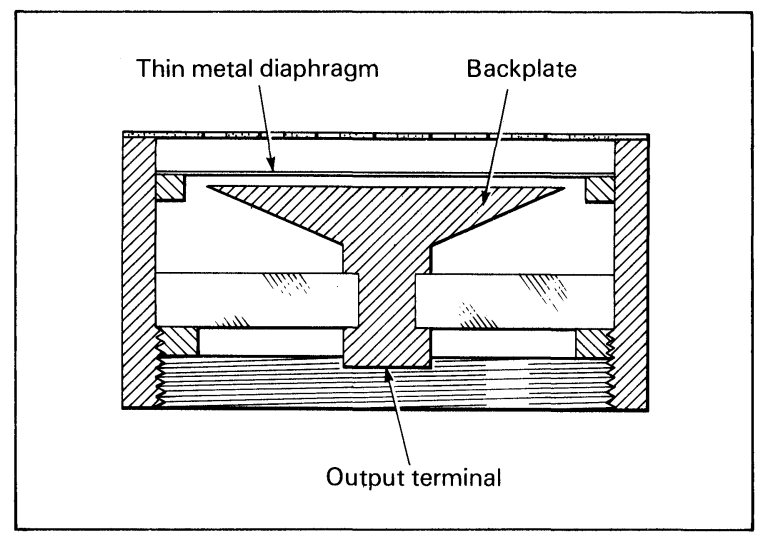

FIGURE 4 Capacitor Microphone 


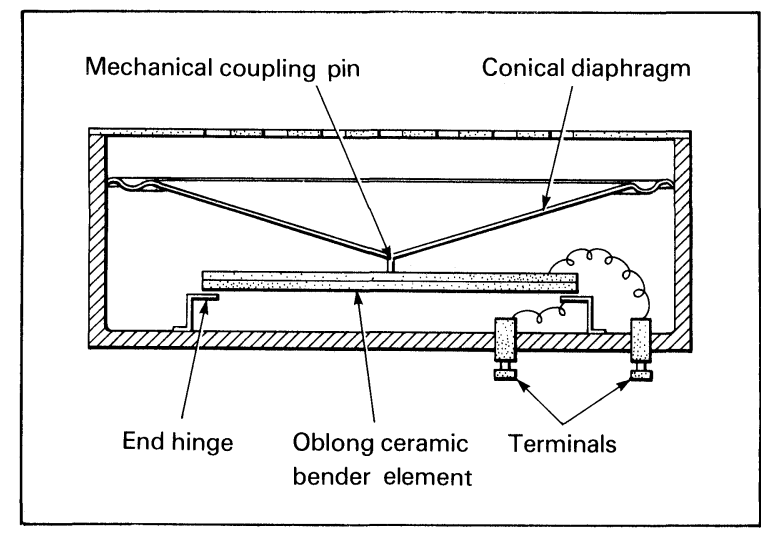

FIGURE 5 Piezo-Ceramic Microphone

mic microphone in which a light metal diaphragm is attached to a ceramic bimorph which produces an electrical output when a force is applied to it. These microphones are high-impedance devices, although not as high as capacitor microphones, and are not suited to arduous conditions because of the risk of damage by mechanical shock.

In recent years, however, piezoelectric plastics have been developed. These materials are relatively tough and may be manufactured cheaply as thin flexible sheets. This raises the possibility of making an extremely simple robust microphone in which the diaphragm and transducer assembly consists solely of sheets of plastic and in which there are no small clearances to be set accurately, or fine wires to be manipulated during manufacture. This type of microphone has been under development at ACRC for some years and several prototypes have been produced for different applications.

\section{PIEZOELECTRIC PLASTIC}

Several plastics show slight piezoelectric activity, but by far the highest activity demonstrated to date is found in polyvinylidene fluoride (PVDF) as reported in 1969 by H. Kawai (ref. 2). PVDF is widely used as wire insulation (under the trade-name KYNAR) and as a chemically resistant coating. The PVDF molecule is a chain of carbon atoms with pairs of fluorine and hydrogen atoms attached to alternate carbon atoms. The molecule can have two different geometrical forms (see Figs. 6 and 7). The preferred form for piezoelectric activity is the $\beta$-phase which has a large dipole momentand hence shows high activity. The proportion of this phase present in the material may be increased by stretching the film during the extrusion process. The film is then rendered piezoelectric by applying a high electric field across the thickness of the sheet and metal electrodes are vacuum-deposited onto it so that electrical connections may be made. A more detailed article on piezoelectric plastics appeared in the 1975 Annual Review.

\subsection{Properties}

The piezoelectric coefficient $d_{31}$ (charge per unit area for a stress in the plane of the film in the direction in which it was stretched during manufacture) is about 20 picocoulombs per newton which is an order lower than that of piezoelectric ceramics but, since the dielectric constant of about 12 is also lower, the avail-

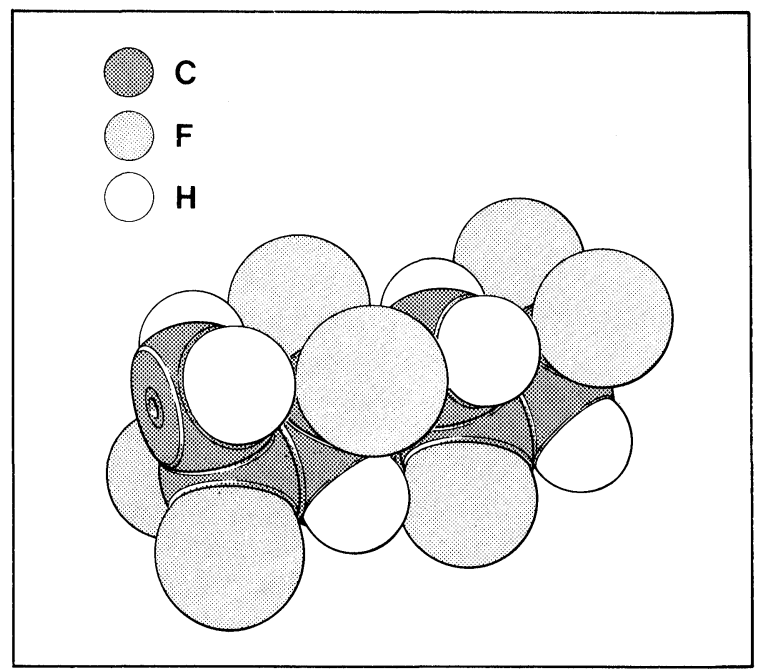

FIGURE 6 a - Phase PVDF

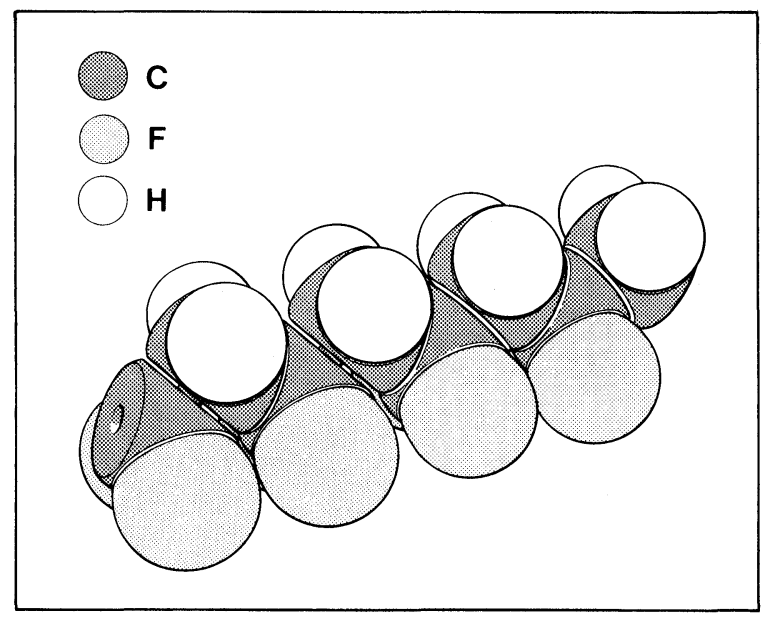

FIGURE $7 \quad \beta$-Phase PVDF 
able voltage output is adequate for practical applications. Ageing tests have shown that provided the PVDF is annealed after poling for a short time at $90-100^{\circ} \mathrm{C}$ the piezoelectric activity is stable enough to meet the requirements specified for military use in extreme environments.

\subsection{A Basic PVDF Microphone Transducer}

The transducer assembly in all the PVDF microphones developed at ACRC has the same basic structure and consists of two sheets of PVDF clamped between two supporting plates, Fig. 8 . The two PVDF sheets behave as a bimorph structure in which one sheet is stretched and the other compressed when sound pressure is incident on them. The polarity of the sheets is arranged so that the electrical outputs reinforce.

A plot of electrical output against frequency for a constant applied sound pressure shows that the frequency response of this structure consists of one peak at resonance and low sensitivity elsewhere. The frequency of this resonance depends on the thickness of the PVDF and the diameter of the holes in the clamping plates. If the resonance is arranged to be near the upper frequency limit, the steep fall in sensitivity can be used to form the upper limit roll-off of the complete microphone. The sensitivity below resonance can be increased by the incorporation of resonant cavities, the details of which will depend upon the required microphone characteristics. The microphone has a highe electrical impedance - a typical assembly appears as a voltage generator in series with a capacitor of $500 \mathrm{pF}$ and so an impedance matching circuit is usually built into the microphone. The input impedance may be used in conjunction with the microphone capacitance to give a low frequency roll-off. There are two classes of microphone: those which are sensitive to changes in pressure and those which respond to the pressure gradient, i.e. the rate of change of pressure with distance, and these will be considered separately.

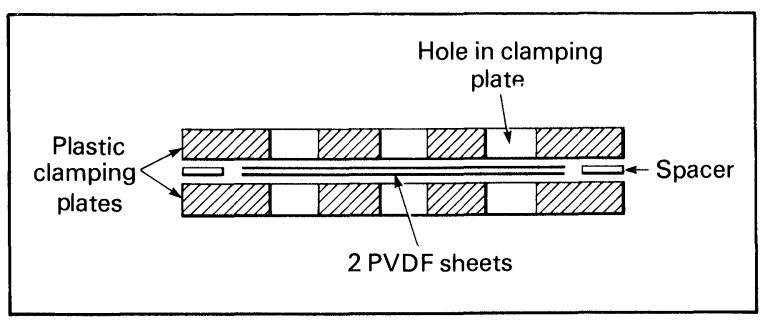

FIGURE 8 Basic PVDF Transducer

\subsection{Pressure-Operated Microphones}

Pressure-operated microphones respond equally to sounds arriving from different distances and directions (neglecting diffraction effects). The PVDF pressure microphone uses the structure shown in Figure 8 with one side of the assembly enclosed by a sealed cavity. (A small vent is provided to allow for barometric pressure equalisation, but the pressure remains substantially constant over the period of a sound wave). The other side of the assembly is enclosed by another cavity which has several holes to allow the entry of the sound. The front cavity acts as a Helmholtz resonator and the cavity and port sizes may be arranged so as to boost the mid-range sensitivity of the microphone. The barometric equalisation hole between the two cavities may be enlarged so that the pressure changes in the rear cavity become significant at low audio frequencies, which gives a sharper low-frequency cutoff.

This type of microphone has been developed for use in telephone handsets and aircrew oxygen masks. A cross-sectional view of a telephone microphone is shown in Fig. 9 and a photograph in Fig. 10. The amplifier circuit in this device is arranged so as to make the microphone resemble a standard carbon microphone electrically and thus enable it to be used as a drop-in replacement within the present telephone system.

\section{NOISE CANCELLING MICROPHONES}

This class of microphone is intended for use in conditions of high background noise. The noise level in military aircraft and armoured fighting vehicles is extremely high, so that use of a pressure microphone would provide a signal with an unusable signal-to-noise ratio whereas a good noise-cancelling microphone can provide an intelligible signal from normal speech when the background noise is so high as to render it impossible to communicate, even over short distances, by shouting. Noise-cancelling microphones rely for their operation on the variation in properties of sound waves with distance from the source. The human voice acts as a point source whose position varies from moment to moment but which is on average some $10 \mathrm{~mm}$ behind the lips. When the sound reaches a boommounted microphone, worn close to the mouth, the wavefronts are spherical. Background noise usually emanates from distant and/or extended sources and the wavefronts can be considered to be substantially planar at the microphone. The pressure gradient in a 


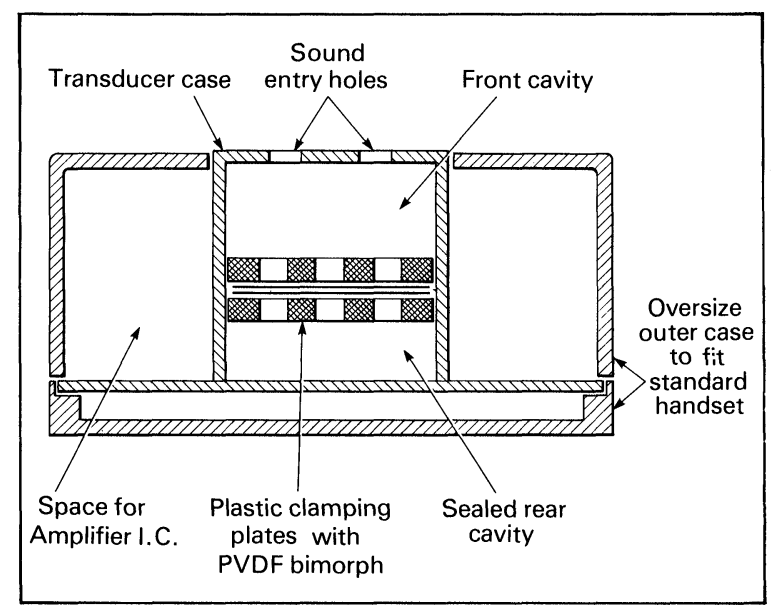

FIGURE 9 PVDF Telephone Microphone

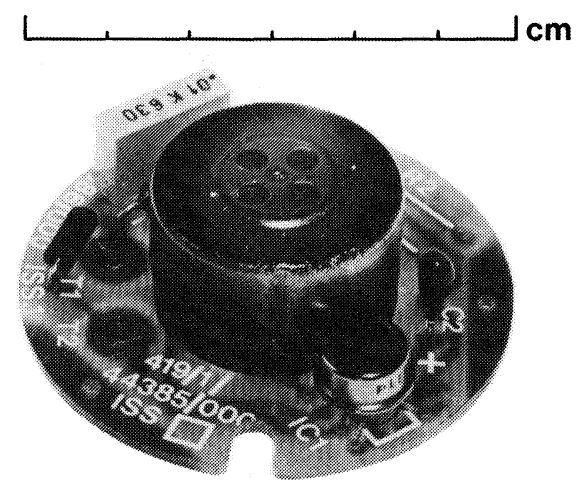

FIGURE 10 Telephone Microphone without outer case

spherical wave is higher than that in a plane wave of equal amplitude, as may be seen from the wave equations:

For a spherical wave $P(t, r)=\left(P_{0} / r\right) \cos k(c t-r)$

where: $P_{0}=$ amplitude, $r=$ distance from point source, $k=2 \pi /$ wavelength, $c=$ speed of sound, $t=$ time.

The gradient,

$$
\mathrm{dP} / \mathrm{dr}=\left(\mathrm{P}_{0} \mathrm{k} / \mathrm{r}\right) \sin \mathrm{k}(\mathrm{ct}-\mathrm{r})-\left(\mathrm{P}_{0} / \mathrm{r}^{2}\right) \cos \mathrm{k}(\mathrm{ct}-\mathrm{r})
$$

The amplitude of the gradient is:

$$
\left(\mathrm{P}_{0} \mathrm{k} / \mathrm{r}\right)\left(1+1 / \mathrm{k}^{2} \mathrm{r}^{2}\right)^{1 / 2}
$$

For a plane wave, $P^{\prime}(t)=P_{0}{ }^{\prime} \cos k(c t-r)$

$$
\text { and the gradient is } \mathrm{dP}^{\prime} / \mathrm{dr}=\mathrm{P}_{0}{ }^{\prime} \mathrm{k} \sin \mathrm{k}(\mathrm{ct}-\mathrm{r} \text { ) }
$$

If the sound pressure of the two waves is equal at the point of measurement, $\mathrm{P}_{0}{ }^{\prime}=\mathrm{P}_{0} / \mathrm{r}$ and therefore the amplitude of the gradient in the plane wave is

$$
\mathrm{P}_{0}{ }^{\prime} \mathrm{k}=\mathrm{P}_{0} \mathrm{k} / \mathrm{r}
$$

From the above, it can be seen that the ratios of the amplitude of the pressure gradients in spherical and plane waves of equal amplitude is $\left(1+1 / \mathrm{k}^{2} \mathrm{r}^{2}\right)^{1 / 2}$ which may be expressed in terms of wavelength, $\lambda$, as $\left(1+\lambda^{2} / 4 \pi^{2} r^{2}\right)^{1 / 2}$. To put this into perspective: the wavelength at $500 \mathrm{~Hz}$ is about $70 \mathrm{~cm}$ and a microphone used close to the mouth is at a radius of about $2.2 \mathrm{~cm}$ so that the ratio of the gradients is 5 to 1 (14 dB). For a practical microphone to show a spherical/plane wave discrimination approaching this theoretical maximum, the microphone should be small since the above analysis assumes an infinitely small microphone.

An even greater discrimination may be obtained by the use of a microphone which responds to the second derivative of pressure. A second-order microphone has been developed which uses four ports arranged in a square and this type of microphone has a discrimination given by:

$$
\begin{aligned}
& \left(1+3 / \mathrm{k}^{2} \mathrm{r}^{2}+9 / \mathrm{k}^{4} \mathrm{r}^{4}\right)^{1 / 2} \\
& =\left(1+3 \lambda^{2} / 4 \pi^{2} \mathrm{r}^{2}+9 \lambda^{4} / 16 \pi^{4} \mathrm{r}^{4}\right)^{1 / 2}
\end{aligned}
$$

A derivation of this expression is given in Ref. 3 . Using the same example as before the ratio of the gradients would be $75.5 / 1(37 \mathrm{~dB})$. Both first-order and second-order microphones have been developed using PVDF transducers.

\section{FIRST-ORDER PRESSURE GRADIENT MICKOPHONE}

These microphones operate by measuring the difference in pressure acting between two sound entry ports on opposite sides of the microphone. The device is made as small as possible since correct operation requires that the size of the microphone be small compared to the shortest sound wavelength of interest. It is also important that the microphone should be acoustically symmetrical, as any inequality in sensitivity between the two ports will increase the output from the background noise. This symmetry is difficult to achieve in the case of an electromagnetic microphone because the presence of the coil and magnet system on one side of the diaphragm must be compensated for whilst keeping the microphone small. The basic PVDF transducer as shown in Figure 8 is an inherently symmetrical structure and is ideally suited for use as a pressure-gradient microphone. 
The frequency response of the basic arrangement is unsatisfactory as it consists of one peak at the resonant frequency with very low sensitivity over most of the audio band. This peak frequency is normally placed near the top of the required range and then the sensitivity below resonance is boosted by the provision of two identical resonant cavities placed symmetrically about the central plane. Damping may then be applied to level out the response, resulting in a smoothly rising response up to the cavity resonance, then a plateau up to the disc resonance and a rapid fall-off above this. The resulting response is suitable for communications use where speech intelligibility is more important than high fidelity: low frequencies contain much background noise and very little wanted information.

Environmental protection is provided by means of thin membranes over the sound entry ports. The amplifier housing forms part of the boom assembly. A cut-away drawing of this microphone is shown in Figure 11. The frequency response of the microphone falls within a typical communications specification and is plotted in Figure 12, which also shows the excellent symmetry of response achieved by this design. The noise discrimination is better than that of the standard military moving-iron microphone in current use, as expected from the improved symmetry.

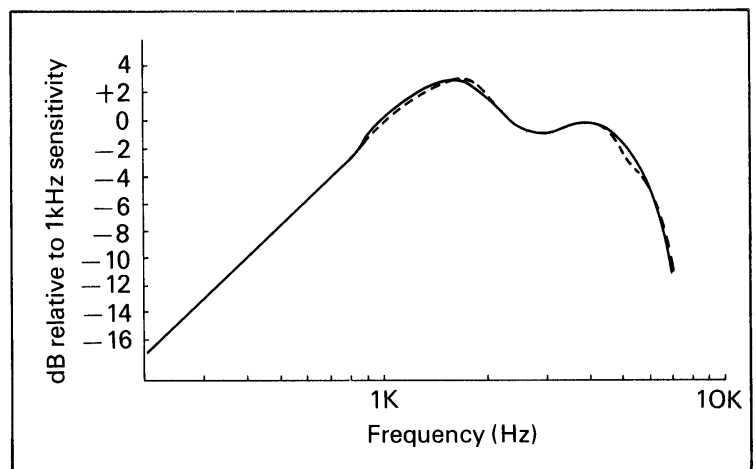

FIGURE 12 Response of first-order microphone to close source

\section{A SECOND-ORDER MICROPHONE}

As mentioned earlier, an even greater difference in sensitivity between spherical and plane waves is produced by a microphone which responds to the second derivative of pressure.

A simple technique for making a second-order microphone using a single transducer was described by Beaverson and Wiggins (Ref. 3). The transducer is situated between two cavities into which sound is admitted through a total four entry ports. In the

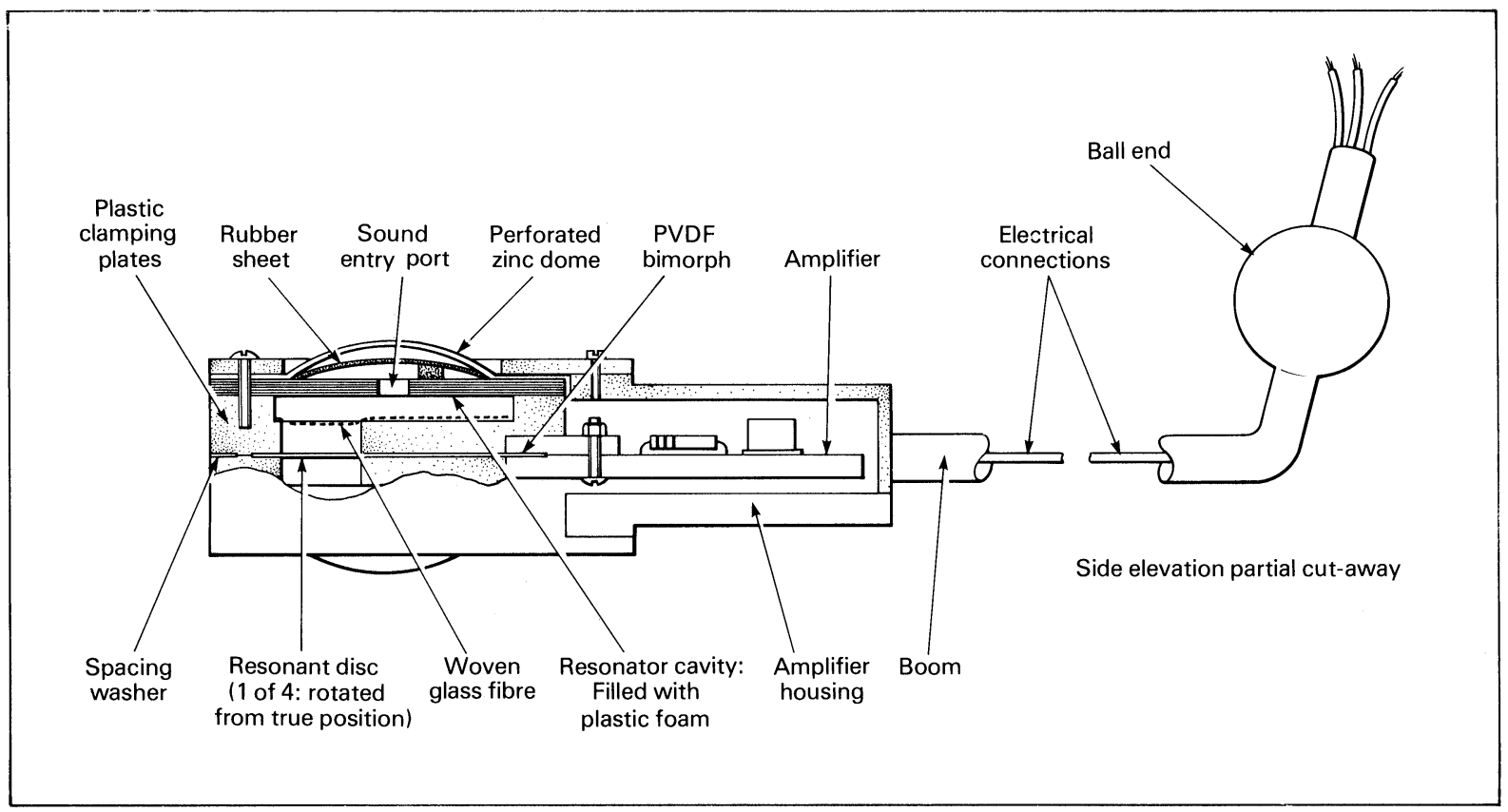

FIGURE 11 Final design sample microphone 


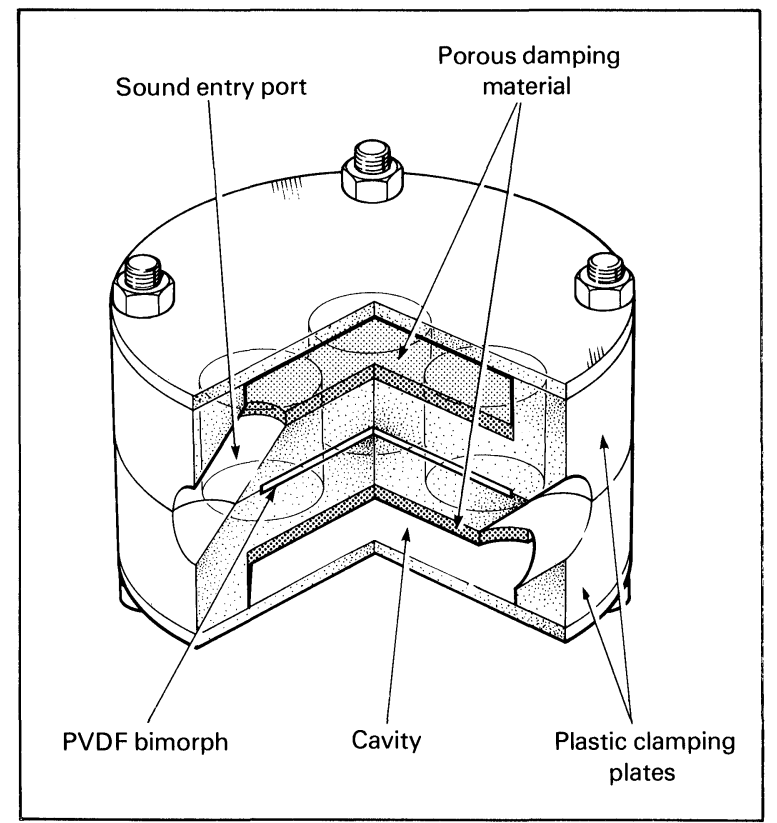

FIGURE 13 Second-order microphone cut-away.

PVDF second-order microphone, the transducer consists of the usual arrangement of edge-clamped resonant discs, and the cavities are arranged so that they act as Helmholz resonators giving a mid-frequency boost in sensitivity. The four ports have their open ends arranged equally spaced around the periphery of the microphone, with each cavity fed by two diametrically opposite ports. A cutaway diagram of this arrangement is shown in Figure 13. The polar response of this microphone is a "four-leaved clover" pattern in the plane of symmetry, with ideally zero pickup on the axis of symmetry.

As with the pressure-gradient microphone, the design exploits the symmetry available with the PVDF transducer. The requirements in this respect are even

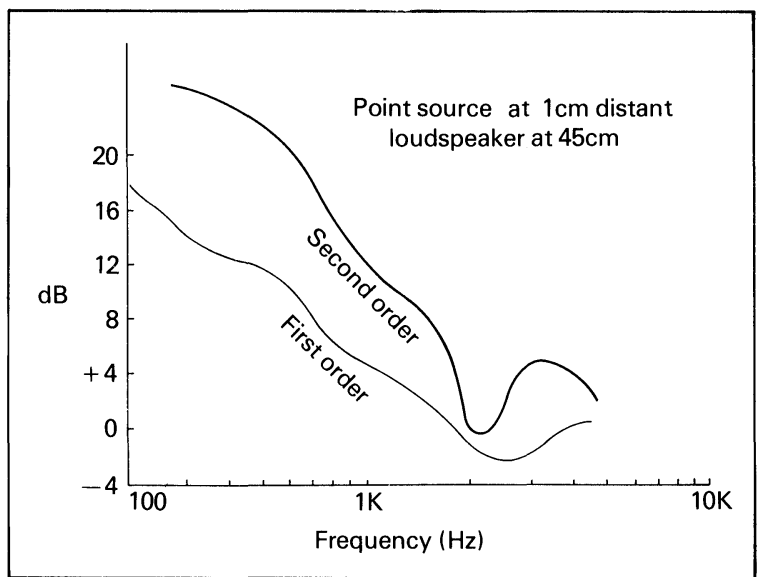

FIGURE 14 Axial noise discrimination for first and second order PVDF microphones.

more stringent with a second-order microphone if the theoretically available performance is to be achieved in practice.

The noise discrimination of first and second order microphones is compared in Figure 14

\section{CONCLUSIONS}

Three piezoelectric plastic microphones have been developed to laboratory prototype stage and their acoustic performances have been shown to have advantages compared with those in current use. They also meet military environmental test requirements.

\section{REFERENCES}

1. Hunnings, British Patent 3647 (Sept. 1878).

2. H. Kawai, Jap. J. Appl. Phys. 8, 975 (1969).

3. W.A. Beaverson and A.M. Wiggins J. Acoust. Soc. Am. 22, 5, 592 (1950). 

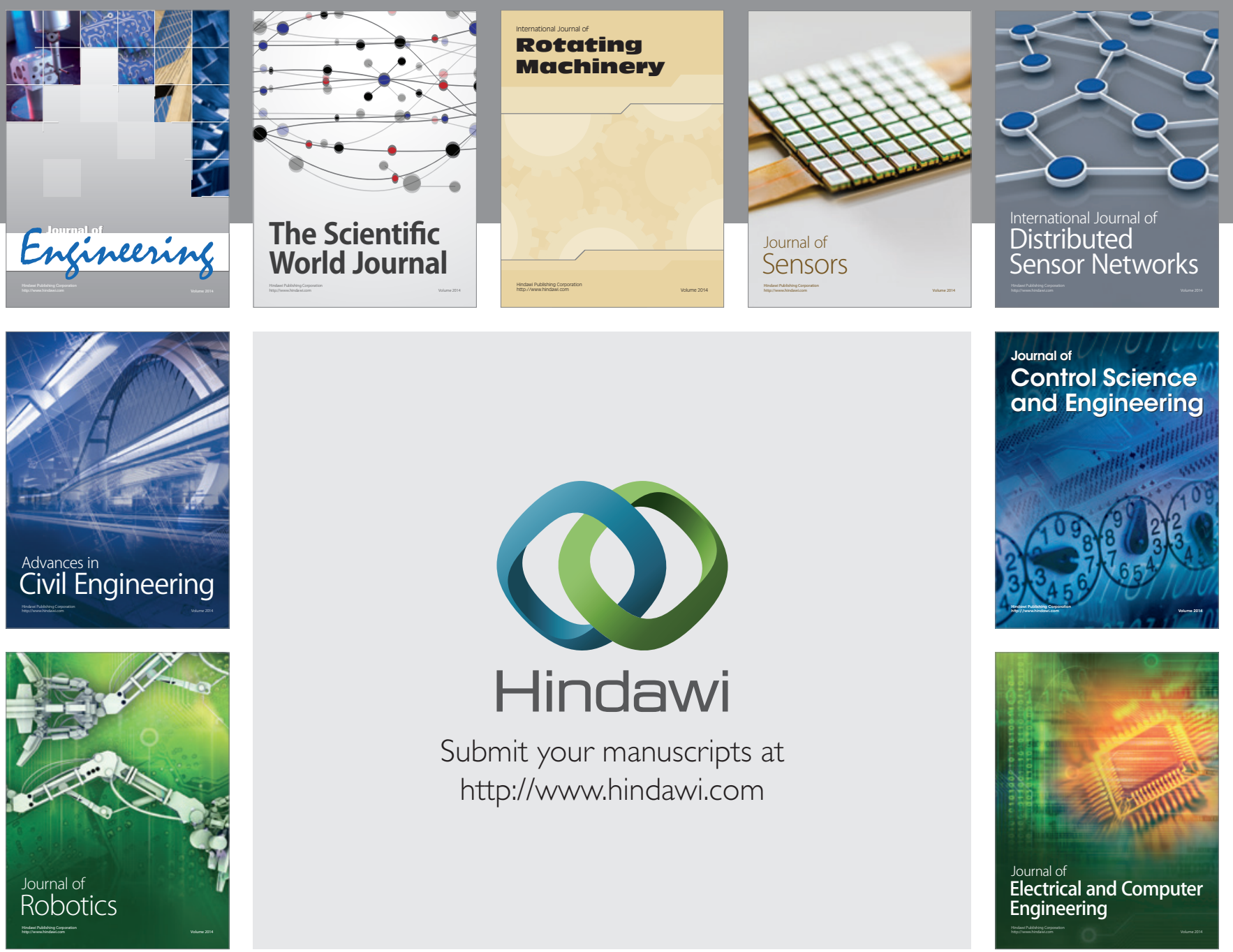

Submit your manuscripts at

http://www.hindawi.com
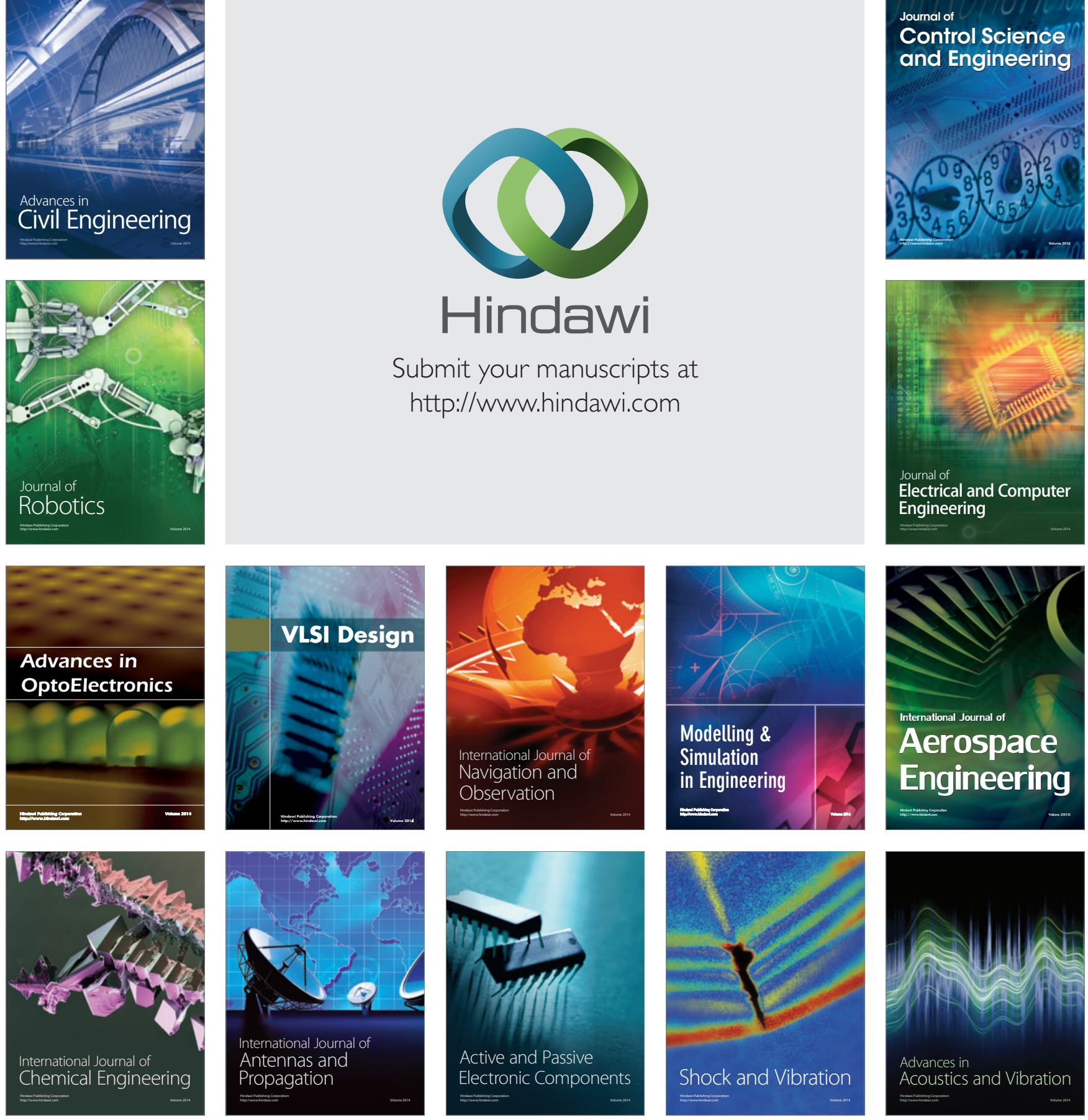